\title{
Transformation Optical User-Friendly Interface for Designing Metamaterials
}

\author{
Pasit Jarutatsanangkoon ${ }^{\mathrm{a}}$, and Wanchai Pijitrojana ${ }^{\mathrm{b}, *}$ \\ Department of Electrical and Computer Engineering, Faculty of Engineering, Thammasat University, \\ Rangsit Campus, Pathumthani 12120, Thailand \\ E-mail: apasit.jaru@gmail.com, bpwanchai@engr.tu.ac.th (Corresponding author)
}

\begin{abstract}
Transformation optics offers a procedure to design the structures of metamaterials to find the material parameters needed in various applications. However, a methodology of a transformation optics is too complicated. To help users who are beginning to study metamaterials and transformation optics, a transformation optical userfriendly interface is developed. The interface is implemented based on the free-form touch transformation. It displays the starting space as a Cartesian grid. As the user touches and moves, the space transforms according to the direction and the intensity of the touch without inputting any equations. By combining various transformation templates, a fully arbitrary transformation can be realized. Transformation templates are created by basic functions such as the ring transformation in the invisibility cloak or the morphing of a half circle into a rectangle in the superlens. The interface provides both the input methods as well as the real-time visualization of the space making it easy and intuitive to design a metamaterial using the transformation optics. The program uses model-view-controller architecture. The connections of each class are presented in a diagram. Three examples from the touch interface are verified by the FDFD simulation.
\end{abstract}

Keywords: Metamaterials, spatial transformation, transformation optics, touch interface.

ENGINEERING JOURNAL Volume 23 Issue 6

Received 28 June 2019

Accepted 12 September 2019

Published 30 November 2019

Online at http://www.engj.org/

DOI:10.4186/ej.2019.23.6.225 


\section{Introduction}

A metamaterial is a material engineered to have a property that is not found in naturally occurring materials. They are made from assemblies of multiple elements fashioned from composite materials such as metals or plastics. The materials are usually arranged in repeating patterns, at scales that are smaller than the wavelengths of the phenomena they influence. Metamaterials derive their properties not from the properties of the base materials, but from their newly designed structures. Their precise shape, geometry, size, orientation and arrangement gives them their smart properties capable of manipulating electromagnetic waves: by blocking, absorbing, enhancing, or bending waves, to achieve benefits that go beyond what is possible with conventional materials. Appropriately designed metamaterials can affect waves of electromagnetic radiation and has led to breakthrough devices with capabilities from superresolution imaging to invisibility. For examples, metamaterials have nanostructured media with hyperbolic dispersion for practical applications from subwavelength resolution imaging in the near and far field [1-5], negative refraction [6-8], invisibility devices [9] and perfect absorbers [10], to nonlinear switching [11]. Metamaterials with microscopic structures are also proposed for applications of the transmission line and microwave [12] such as the ultra wideband antenna [13-18], the leaky-wave antenna [19, 20], the resonator [21], the dual-band RFID antenna [22], the coupled-line phaser [23], the magnetless nonreciprocal gyroscopic metasurface [24]. Furthermore, metamaterials are used for an acoustic transformation media [25], or a two-layered isotropic thermal media [26] in a manner not observed in the bulk materials.

However, appropriately designed structures of metamaterials are conventionally complicated to be designed and optimized as outlined by Veselago et al. [27] and Li et al. [28]. Recently, transformation optics (TO) offers a great procedure to design the structures of metamaterials to find the material parameters needed in various applications, especially to design the anisotropic media. Anisotropic media exhibit interesting optical properties and are used to create optical devices such as the invisibility cloak [9], the superlens and the waveguide bend [29,30].

The transformation optics theory which starts from the designing of the EM field pattern rather than the trial and error of material parameters tremendously assists in the metamaterial design process. By transforming the geometry of the empty virtual space and linking it to the metamaterial-filled physical space, ones can find the material parameters from the spatial transformation [31]. The spatial transformation can be applied using various method such as a closed-form mathematical function [9, 29-31], a solution from differential equation with boundary conditions [32-35], resulting in a well-defined virtual space. The wellknown applications of the methods are the invisibility cloak by Pendry et al. [9] and Schurig et al [36] and the wave collimator by Kwon and Werner [37]. However, in these cases, transformations were described by the closed-form expressions, which were derived from an analytical approach. This approach, while perfectly describing the transformed coordinates, relies on the intuition and the careful analysis of the target application. An expression might not be able to describe every arbitrary transformation. To overcome this, Laplace's equation [38, 39], Poisson's equation [40], and the Helmholtz's equation [41] are proposed to solve for the arbitrary transformation according to the boundary conditions. Furthermore, in these works, a commercial finite element software was used to simulate the resulting material. Recently, a full numerical toolchain was developed by Berry et al. [42]. This tool adapts the discrete nature of the anisotropic finitedifference frequency-domain (AFDFD) method [43, 44] into the solving of Laplace's equation. The resulting material parameters can also be exported and used in other solvers. However, these methods rely on knowing the boundary conditions of space to solve for the curvature of space between the boundaries. Another method of designing an optical device is to combine the geometrical optics with the transformation optics [45]. This method utilizes the well-known properties of the conventional geometrical devices for creating a more enriching optical design. However, the design process is complicated which may be daunting to some.

To help users who do not have extensive knowledge of transformation optics but want to use the transformation optical methods to design metamaterials for applications, in this paper, we propose userfriendly interface with transformation optics for designing the free-form user interface with a touch interface. The objective is to allow users to easily design a transformation without knowing the underlining transformation function. The program uses the model-view-controller architecture. The model class represents the working space and contains methods for the spatial transformations and the calculation of material parameters. The view class provides the visualization of the space, and the user interfaces for adjusting the transformation parameters. The controller class communicates between the view and the model by passing the transformation from the view to the model and sending the transformed space back 
to the view. The resulting material parameters are imported into the FDFD program for verification [43, 44]. The program can be extended by providing the mathematical functions to the model and the user interfaces to the view. Three examples of spatial transformations are provided to verify the software.

\section{Program Architecture}

The program is developed on the iOS platform using Swift programming language. The advantages of this platform are the wide-variety user interfaces available for creating a program and the intuitive touch interfaces for interacting with the space. The program utilizes the model-view-controller architecture. The model handles the logic and the calculation of the program. The view contains the user interface for user input and visualization. The controller handles the communication between the model and the user interface.

\subsection{Model}

From the theory of transformation optics, the effect of material on the propagation of electromagnetic wave is the same as the effect of the geometry of the space that the wave travels on [31]. In a real world, the space is Euclidean which means the unit distances in all direction have the same effective length. We represent the Euclidean space by a Cartesian grid as shown in Fig. 1.

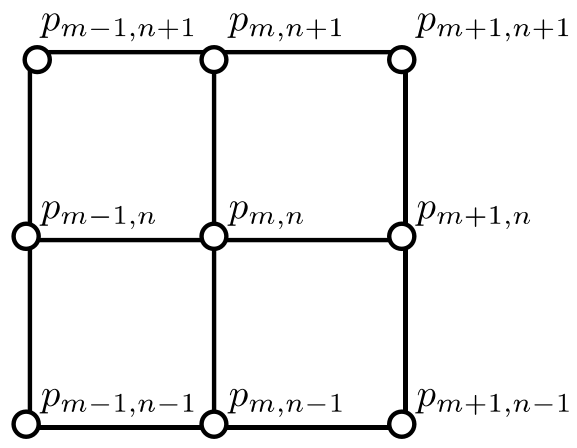

Fig. 1. The starting Euclidean space represented by a Cartesian grid. The positions of the grid points change according to the transformation applied. These changes can be converted into the material parameters.

The positions of the grid points are stored in an array $\mathrm{P}$ with the first index corresponds to the $\mathrm{x}$ position and the second index corresponds to the y position. When the transformation is applied, the points shift according to the user input. The new transformed space represented by an arbitrary grid is shown in Fig. 2.

Each transformation has different characteristics but we can categorize them into two groups. The first group is the in-place transformation where the transformation is applied directly to the current space thus creating no new space as shown in Fig. 2. The second group is the embedded transformation where a new curved space replaces a portion of the current space as shown in Fig. 3.

To differentiate between the applicable points and the discarded points, we create a Boolean array point_is_used which is reassigned every time a new space is created. The program also requires a space array to store all spaces created during the process. This can be done by storing instances of spaces sequentially and combining them during the visualization. The space array is stored in the master controller. Therefore, it can be accessed by any view controller. The mechanism of the in-place transformation and the embedded transformation are shown in Fig. 4 and Fig. 5 respectively. 


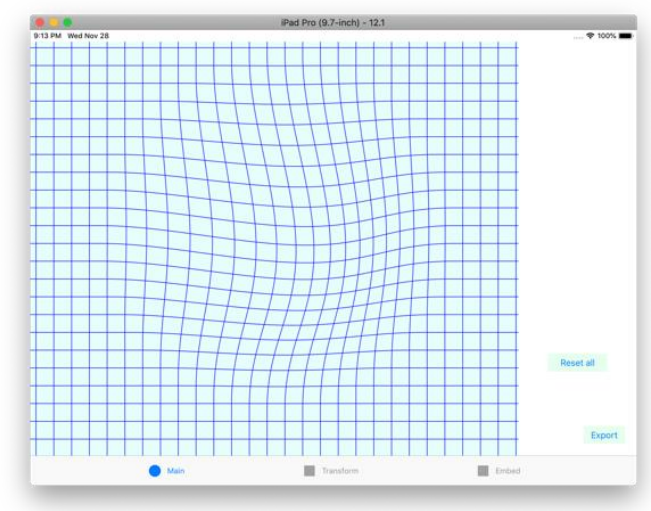

Fig. 2. An example of a transformed space represented by a curve grid. This is an in-place transformation. The space, including all electromagnetic waves that travel through that region of space, is shifted. Therefore, the apparent paths of the waves are along the curve grid.

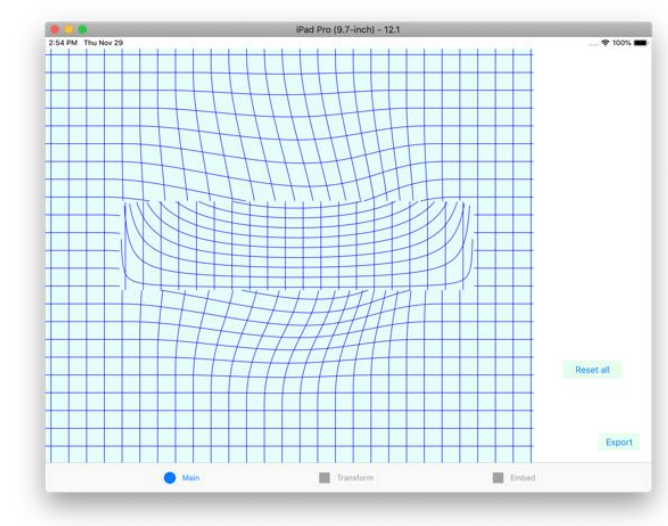

Fig. 3. A superlens space is embedded into the current space. The embedded transformation is analogous to the placement of a lens in the traditional optical system.

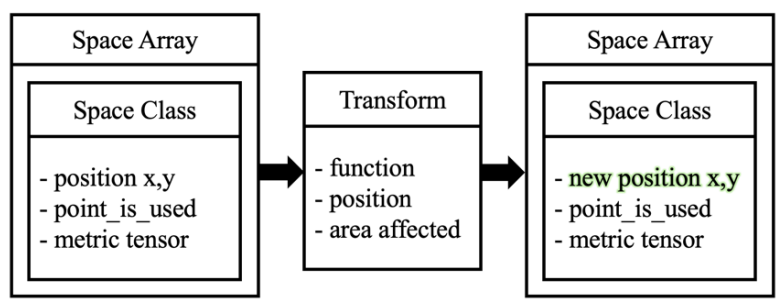

Fig. 4. The in-place transformation. The space is transformed resulting in the new grid positions. No new space is created.

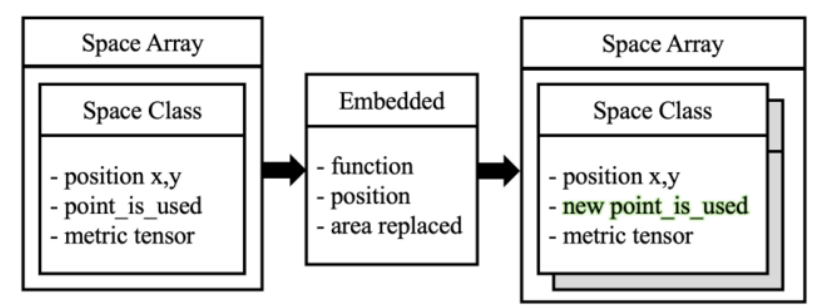

Fig. 5. The embedded transformation. A new space replaces an area in the current space. The Boolean point_is_used is updated. 
The steps of applying the transformation are as followed. Each transformation acts on a finite region in space. Therefore, the first step is to get only the points within that region. This can be done by looping over all points in all spaces and selecting only the points that are applicable. Then the transformation function will move the points accordingly. This can be done simply by looping over all points. To fine-tune the transformation, one can specify the exact value of the transformation parameters. Multiple transformations can be applied until the desire geometry has reached. The last step is to calculate the material parameters from the grid points with the following equations [46].

$$
\varepsilon^{\prime}=\mu^{\prime}=G^{-1}\left[\operatorname{det}\left(G^{-1}\right)\right]^{-\frac{1}{2}}
$$

where $\varepsilon^{\prime}, \mu^{\prime}$ are the relative permittivity and permeability of physical space respectively, $G^{-1}$ is the inverse metric tensor which can be calculated from the point coordinates with the following equations [46].

$$
\begin{gathered}
G^{-1}=\left[\begin{array}{ll}
g_{11} & g_{12} \\
g_{12} & g_{22}
\end{array}\right] \\
g_{11}=\frac{1}{4} \times\left[\left(p_{m+1, n}^{1}-p_{m-1, n}^{1}\right)^{2}+\left(p_{m, n+1}^{1}-p_{m, n-1}^{1}\right)^{2}\right] \\
g_{12}=\frac{1}{4} \times\left[\left(p_{m+1, n}^{1}-p_{m-1, n}^{1}\right)\left(p_{m+1, n}^{2}-p_{m-1, n}^{2}\right)+\left(p_{m, n+1}^{1}-p_{m, n-1}^{1}\right)\left(p_{m, n+1}^{2}-p_{m, n-1}^{2}\right)\right] \\
g_{22}=\frac{1}{4} \times\left[\left(p_{m+1, n}^{2}-p_{m-1, n}^{2}\right)^{2}+\left(p_{m, n+1}^{2}-p_{m, n-1}^{2}\right)^{2}\right]
\end{gathered}
$$

where $p^{1}, p^{2}$ are the first and second coordinates of the points. Finally, the model exports the resulting metric tensor, $\mathrm{x}$ position, y position and the point_is_used to the simulation program for verification.

\subsection{View}

The view class contains the user interface for designing and visualizing the space. The view hierarchy is shown in Fig. 6. The root view has a tab bar which connects to the main view, the transform collection view and the embedded collection view. Both collection views show the available templates. Clicking on any template brings up the corresponding customization view. The customization view displays the current space with the outline of a new transformation to be applied.

As an example, the ring customization view is the view for designing the invisibility cloak by radially pushing the space outward from a single point. This creates a new space in the inner circle which an electromagnetic wave cannot propagate through but rather propagate around. The view contains sliders and text fields for the radius ratio, the outer radius, the x position and the y position as shown in Fig. 7. As the parameters are adjusted, the grid lines change to visualize the new space. For a high smoothness of the transformation animation, only the points at grid lines are transformed during the customization process. Clicking the apply button will transform all points in space.

For an embedded transformation, we use a superlens as an example. A superlens is originally a halfcircle Euclidean space as shown in Fig. 8a which will be transformed into a non-Euclidean space as shown in Fig. 8b. A line source situated at the center of this half-circle will generate a cylindrical wave which has its wavefront parallel to the circular edge as shown in Fig. 9a. The transformation changes this circular edge into a straight edge as shown in Fig. 9b. The wavefront will still be parallel to the edge. Thus, the wave propagated from this new rectangular non-Euclidean space will be a plane wave. The superlens customization view provides the sliders to adjust the thickness, radius, and position of the superlens. There is also a progress slider to show the gradual transformation of the virtual half-circle space into the physical rectangle space. 


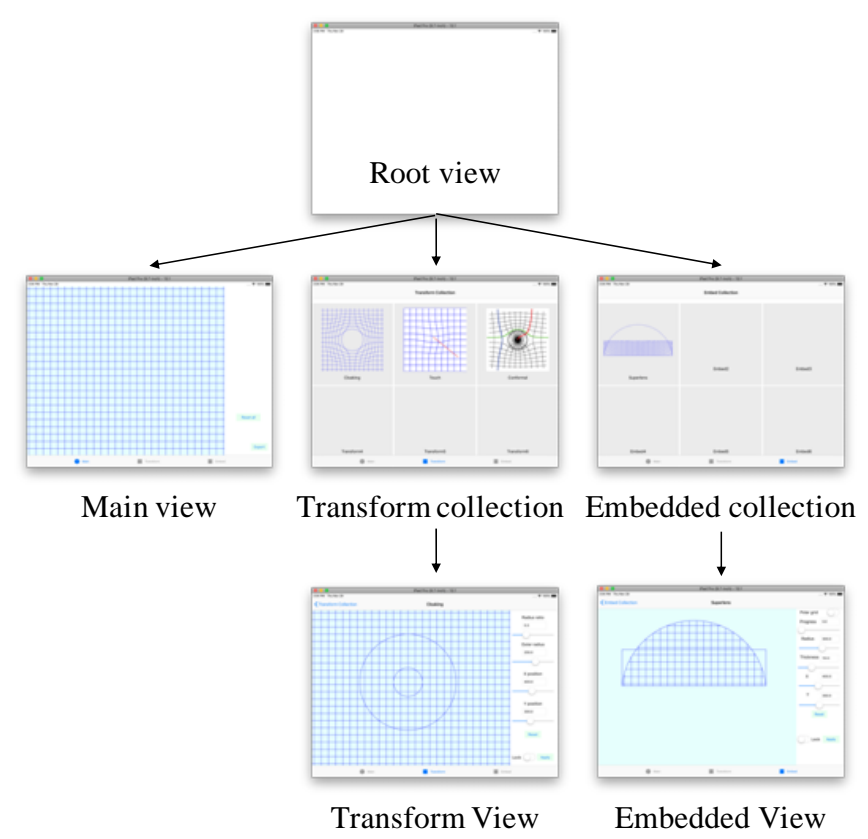

Fig. 6. The view hierarchy of the program. The root view has a tab bar which connects to the main view, the transform collection view and the embedded collection view. Selecting any template will bring up the customization view for fine-tuning the transformation. After applying the transformation, the main view will redraw the current combined space.

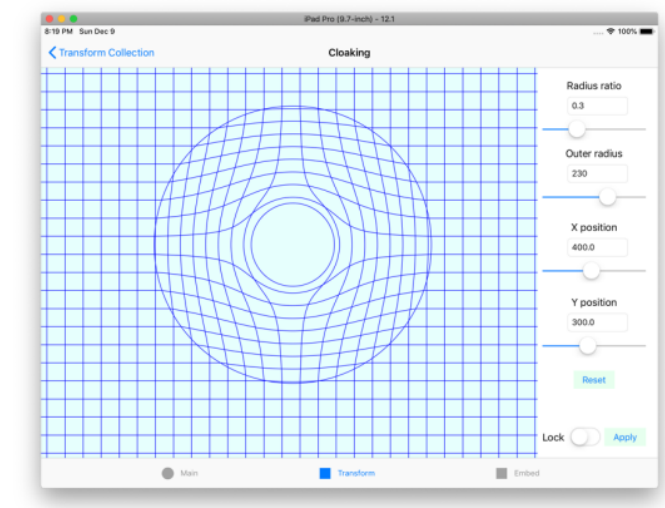

Fig. 7. The ring customization view. A user can adjust the radius ratio, the outer radius, the $\mathrm{x}$ position and the $y$ position. As the user moves the slider or types in a number, the view will redraw the space for a realtime visualization. Clicking apply will confirm the change. 

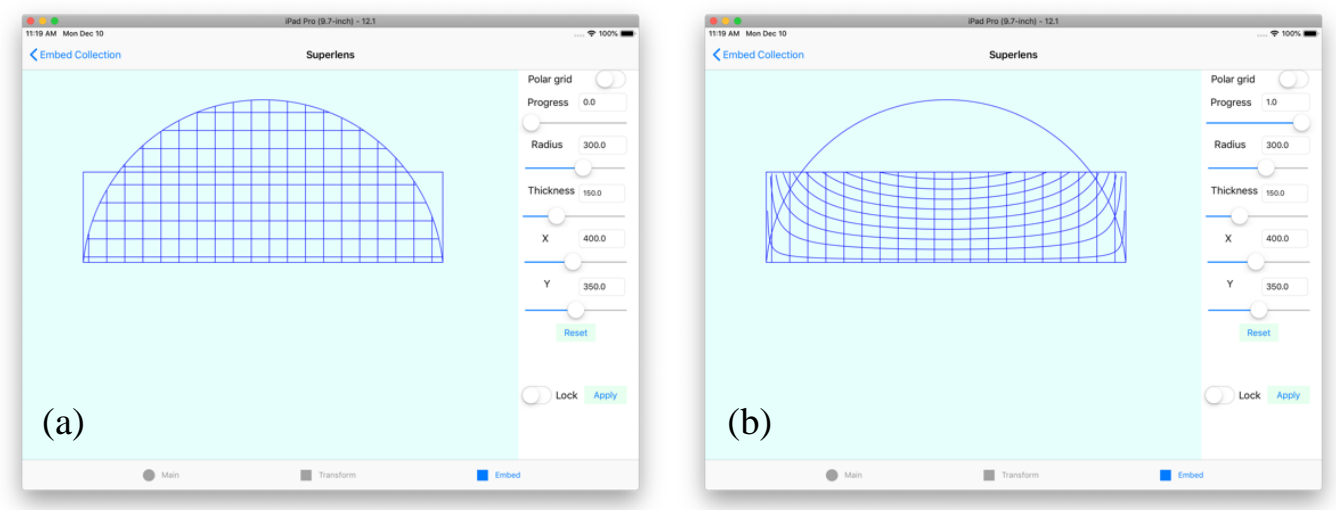

Fig. 8. The superlens customization view in a Cartesian grid. (a) The starting space is a half-circle Euclidean space. (b) The resulting space is the original half-circle space which has been compressed around the center and stretched at both edges.

These two examples demonstrate the ease of customization and visualization of the transformed space. There is no transformation function for users to write nor the imagining of the resulting space. The transformation optics is implemented visually and intuitively.
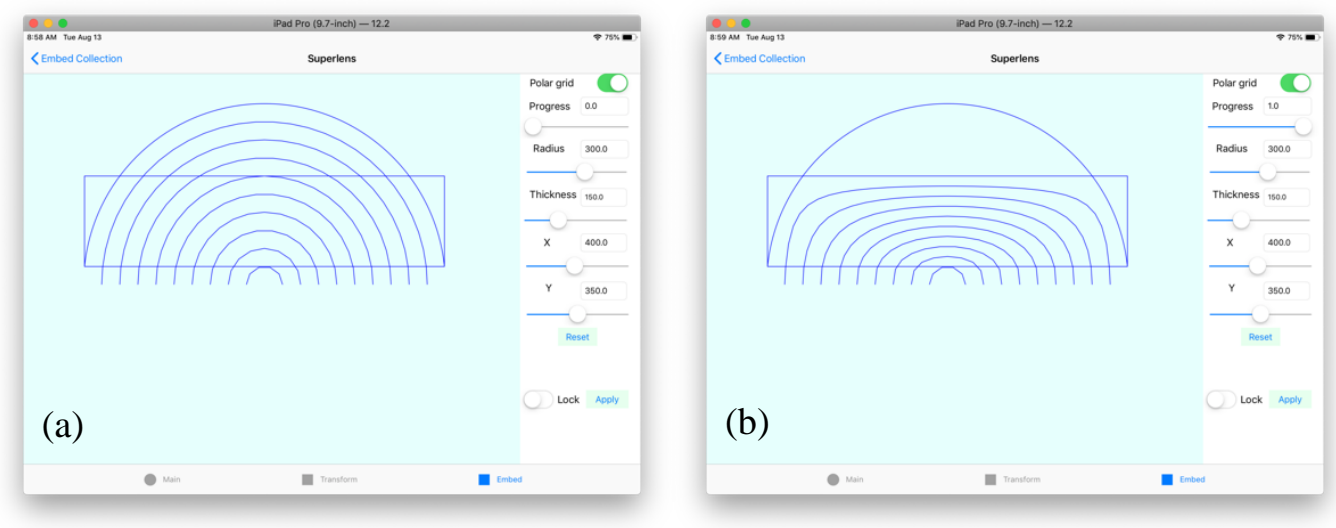

Fig. 9. The superlens customization view in a polar grid. (a) The starting space is a half-circle Euclidean space where the electromagnetic wave propagates radially from the center of the half circle. The wavefront is parallel to the circular edge. (b) The resulting space is the original half-circle space which has been compressed around the center and stretched at both edges. The wavefront is still parallel to the edge resulting in a plane wave.

\subsection{Controller}

The controller connects the space model to the view so that they are independent of each other. The architecture of the entire program is shown in Fig. 10. The main view is connected to the master controller. After the program launches, the master controller initializes the space array which contains one Cartesian space initially. Once user chooses the customization view, the corresponding view controller is loaded. View controller will receive the inputs through text fields, sliders or touch events and send them to the space model to transform. The model will update its temporary grid and the point_is_used Boolean and draw a new grid path. Clicking on the apply button will transform the rest of the points. More transformations can be added in the same way. The export button will trigger the model to calculate the metric tensor and write it along with the positions and the Boolean of each metric to a file. The master controller stores the space array which can be accessed by any view controller. It also handles the addition 
of space into the array as explained in Section 2.1. The design program outputs the space description in the form of the metric. The next step is to import the metric into the simulation program to verify the result.

\subsection{FDFD Simulation}

The metric tensor from the design program will be converted to the material parameters as explained in Section 2.1. Using the material parameters, we solve the Maxwell's equations for the resulting EM field pattern. The numerical method used is the Finite-Difference Frequency-Domain (FDFD) method [43, 44]. The reasoning for choosing FDFD over Finite Element Method (FEM) is the meshing. FEM utilizes a triangular mesh which is more complicated to implement than the uniform rectangular grid of FDFD. In addition, the result from the design program is already in a rectangular grid, albeit slightly curved. Therefore, we interpolate the material parameters into a Cartesian grid before assigning them to the FDFD grid. The results from the simulation will be compared to the designs from the design program. In the next section, three examples are given to verify the program.

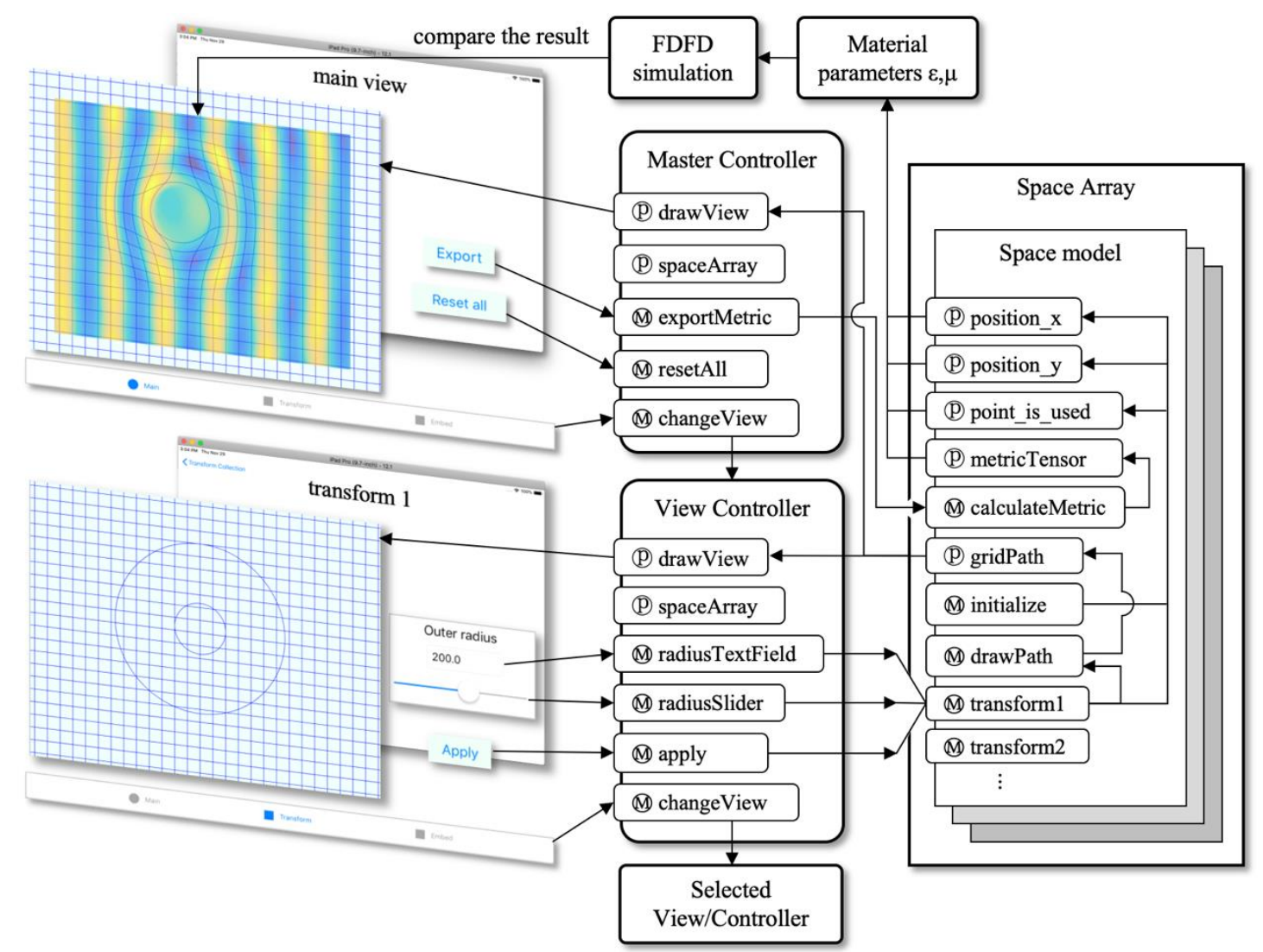

Fig. 10. The program architecture showing the views, the controllers and the model. The symbol (P) means a property and $(\mathrm{M})$ means a method. The program starts at the main view which has a tab bar to change to the transform customization view. The transform 1 view sends the input to its view controller and receives a new space to draw. The view controller asks the space model to perform all calculations making it easy to update the model. If a new space is embedded into the current space, it will be added to the space array. Once the user finishes the design and clicks the export button, the master controller asks the space model to calculate the metric tensor. The master controller exports the metric tensor along with the position $\mathrm{x}, \mathrm{y}$ and the Boolean point_is_used to the FDFD simulation program. Finally, the resulting electromagnetic field pattern is compared to the design in the main view for verification. 


\section{Transformation Examples}

\subsection{Touch Transformation}

The first example is the touch transformation in which the space is elastically pulled with a touch gesture [46] as shown in Fig. 11. Originally, a plane wave propagating in the $\mathrm{x}$ direction will keep propagating in a straight line. The wavefront is parallel to the vertical lines. Once the touch transformation is applied, the grid becomes curved. The wavefront is also curved in the same manner. The design program then generates the material parameters as shown in Fig. 12. The material parameters are used in the FDFD algorithm to find the resulting EM field pattern which is overlaid in Fig. 11. The simulation result matches the design.

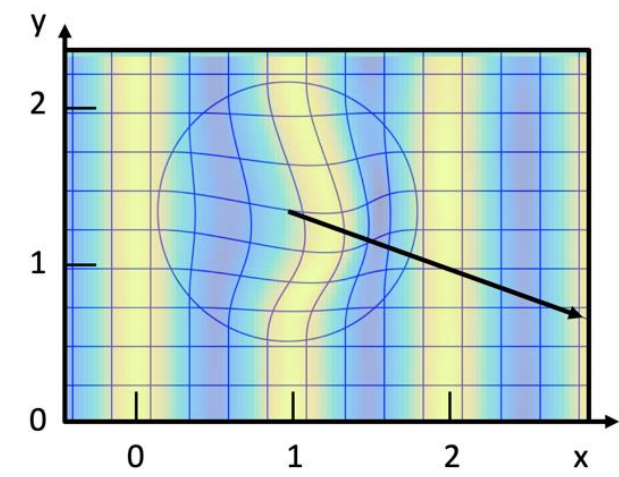

Fig. 11. The touch transformation example. A plane wave is traveling in the positive $\mathrm{x}$ direction. The wavefront matches with the curved grid from the touch transformation. The distance is in a unit wavelength.
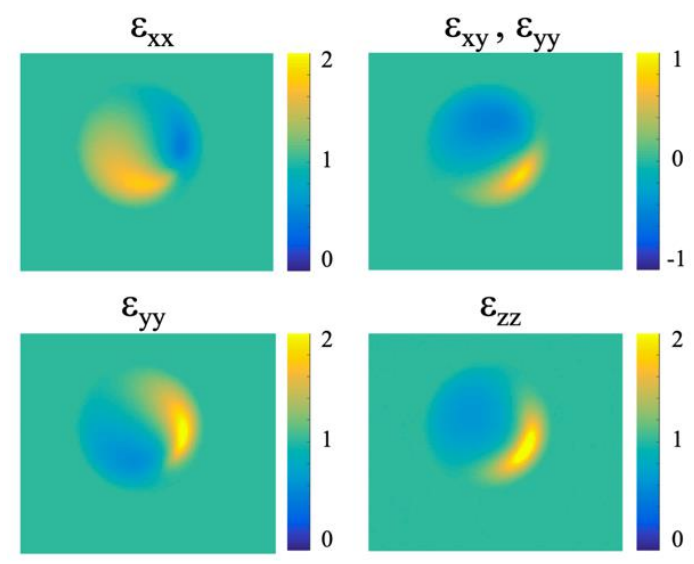

Fig. 12. The material parameters from a touch transformation. The relative permeability tensor $\mu$ is the same as the relative permittivity tensor $\varepsilon$.

\subsection{Ring Transformation}

The ring transformation expands a center point into an inner circle [36]. All points are pushed radially into the ring region as shown in Fig. 13. In this example, the outer radius is 2, the radius ratio is 0.3 making the inner radius equal to 0.6 . The center is at $(2.5,2.5)$. The distance is in a unit wavelength. The incident wave is a plane wave propagating in the $\mathrm{x}$ direction. The wave inside the invisibility cloak will propagate in the direction of the horizontal line, that is, the wave is propagating around the inner circle. The wavefronts will be the same as the curved vertical lines. The material parameters from the design program are shown in Fig. 14. The resulting EM field pattern is overlaid in Fig. 13. The simulation shows that the wave will propagate around the inner region as designed. 


\subsection{Superlens transformation}

The superlens transforms a cylindrical wave into a plane wave [29] as explained in Section 2.2. In this example, the incident wave is the cylindrical wave centered at the center of the half-circle space $(0,-0.3)$. The half circle has a radius of 3 and an offset of 0.3. The resulting superlens has a thickness of 1.5. The distance is in a unit wavelength. The superlens is embedded in the Euclidean space as shown in Fig. 15. The superlens space is compressed vertically at the center and gradually expanded as we move further horizontally from the center. At the edge of the superlens, the space is greatly expanded as evident by the grid line in Fig. 15. The material parameters from the design program are shown in Fig. 16. The simulation result shows the transformation of the cylindrical wave into the plane wave which corresponds to the design of the superlens.

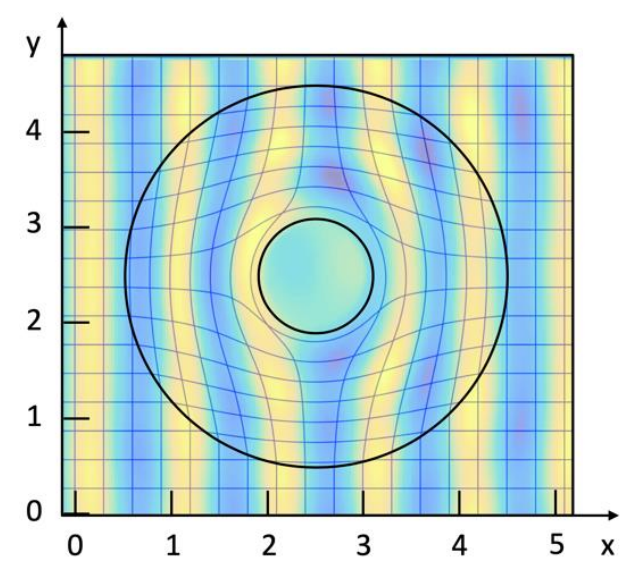

Fig. 13. The ring transformation example. A plane wave is traveling in the positive $\mathrm{x}$ direction. The wavefront matches with the curved grid from the ring transformation. The wave propagates around the inner circle rendering the object inside invisible. The distance is in a unit wavelength.

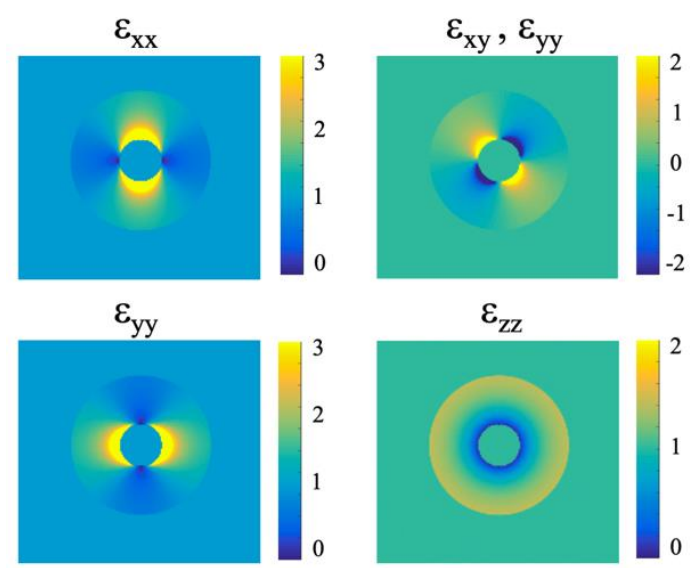

Fig. 14. The material parameters from a ring transformation. The relative permeability tensor $\mu$ is the same as the relative permittivity tensor $\varepsilon$. 


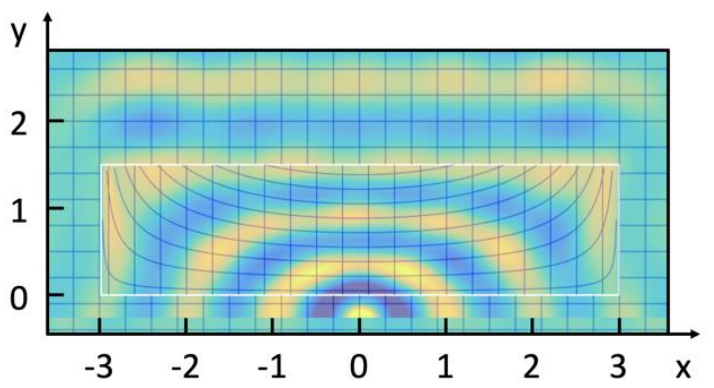

Fig. 15. The superlens transformation example. A line source is located at $(0,-0.3)$. The cylindrical wave enters the superlens at $\mathrm{y}=0$ and gradually transforms into a plane wave and exits the superlens at $\mathrm{y}=1.5$. The distance is in a unit wavelength.

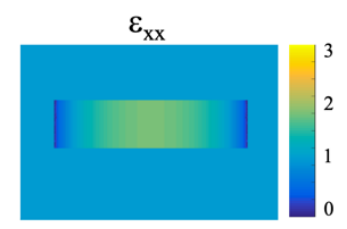

$\varepsilon_{\mathrm{yy}}$

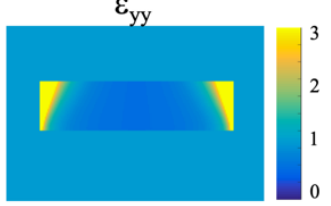

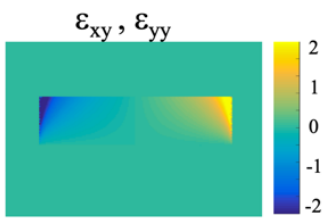

$\varepsilon_{\mathrm{zz}}$

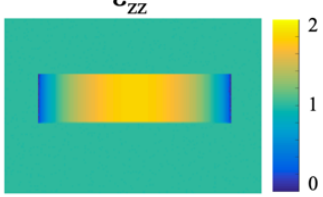

Fig. 16. The material parameters from a superlens transformation. The relative permeability tensor $\mu$ is the same as the relative permittivity tensor $\varepsilon$.

To add more transformation templates, user can add transformation functions to the space model and create the corresponding customization views and view controllers.

\section{Conclusion}

A program with touch interface is developed for designing metamaterial using transformation optics with an arbitrary spatial transformation. It uses a model-view-controller architecture which is easily expandable. The program implementation is discussed in great details. Each transformation has a corresponding view for customization and a transformation function in the model. The touch interface allows for an intuitive application of the transformation. Transformations are stored in a curved grid and are easily converted to the material parameters which can be used to verify the design using FDFD method. Three results from the FDFD simulation verify the correctness of the program.

\section{Acknowledgements}

This work receives a funding from Thailand Research Fund (TRF) and Thammasat University (TU) through the Royal Golden Jubilee (RGJ) Ph.D. Programme.

\section{References}

[1] N. Fang, H. Lee, C. Sun, and X. Zhang, "Sub-Diffraction-Limited Optical Imaging with a Silver Superlens," Science, vol. 308, no. 5721, pp. 534-537, 2005.

[2] Z. Jacob, L. V Alekseyev, and E. Narimanov, "Optical hyperlens: Far-field imaging beyond the diffraction limit," Opt. Express, vol. 14, no. 18, pp. 8247-8256, Sep. 2006.

[3] Z. Liu, S. Durant, H. Lee, Y. Pikus, N. Fang, Y. Xiong, C. Sun, and X. Zhang, "Far-field optical superlens," Nano Lett., vol. 7, no. 2, pp. 403-408, Feb. 2007. 
[4] Z. Liu, H. Lee, Y. Xiong, C. Sun, and X. Zhang, "Far-field optical hyperlens magnifying subdiffraction-limited objects," Science, vol. 315, no. 5819, p. 1686, 2007.

[5] I. I. Smolyaninov, Y.-J. Hung, and C. C. Davis, "Magnifying Superlens in the Visible Frequency Range," Science, vol. 315, no. 5819, pp. 1699-1701, 2007.

[6] V. G. Veselago, "The electrodynamics of substances with simultaneously negative values of $\varepsilon$ and $\mu$," Phys. Usp., vol. 10, no. 4, pp. 509-514, 1968.

[7] V. M. Shalaev, "Optical negative-index metamaterials. Nat. Phot. 1, 41," Birck NCN Publ., vol. 1, 2007.

[8] S. Foteinopoulou, E. N. Economou, and C. M. Soukoulis, "Refraction in media with a negative refractive index," Phys. Rev. Lett., vol. 90, no. 10, p. 107402, Mar. 2003.

[9] J. B. Pendry, D. Schurig, and D. R. Smith, "Controlling electromagnetic fields," Science, vol. 312, no. 5781, pp. 1780-2, 2006.

[10] N. I. Landy, S. Sajuyigbe, J. J. Mock, D. R. Smith, and W. J. Padilla, "Perfect metamaterial absorber," Phys. Rev. Lett., vol. 100, no. 20, p. 207402, May 2008.

[11] M. Shoaei, M. K. Moravvej-Farshi, and L. Yousefi, "All-optical switching of nonlinear hyperbolic metamaterials in visible and near-infrared regions," J. Opt. Soc. Am. B, vol. 32, no. 11, pp. 2358-2365, Nov. 2015.

[12] C. Caloz and T. Itoh, Electromagnetic Metamaterials, Transmission Line Theory and Microwave Applications. Wiley-IEEE Press, 2005.

[13] M. Alibakhshi-Kenari, M. Movahhedi, and H. Naderian, "A new miniature ultra wide band planar microstrip antenna based on the metamaterial transmission line," in 2012 IEEE Asia-Pacific Conference on Applied Electromagnetics (APACE), 2012, pp. 293-297.

[14] M. Alibakhshi-Kenari, M. Naser-Moghadasi, R. A. Sadeghzadeh, B. S. Virdee, and E. Limiti, "Periodic array of complementary artificial magnetic conductor metamaterials-based multiband antennas for broadband wireless transceivers," IET Microwaves, Antennas Propag., vol. 10, no. 15, pp. 1682-1691, 2016.

[15] M. Alibakhshi-Kenari, M. Naser-Moghadasi, R. Ali Sadeghzadeh, B. Singh Virdee, and E. Limiti, "New Compact antenna based on simplified CRLH-TL for UWB wireless communication systems," Int. J. RF Microw. Comput. Eng., vol. 26, no. 3, pp. 217-225, Mar. 2016.

[16] M. Alibakhshi-Kenari, M. Naser-Moghadasi, R. A. Sadeghzadeh, B. S. Virdee, and E. Limiti, "Traveling-wave antenna based on metamaterial transmission line structure for use in multiple wireless communication applications," AEU - Int. J. Electron. Commun., vol. 70, no. 12, pp. 1645-1650, 2016.

[17] M. Alibakhshi-Kenari, M. Naser-Moghadasi, R. A. Sadeghzadeh, B. S. Virdee, and E. Limiti, "Bandwidth extension of planar antennas using embedded slits for reliable multiband RF communications," AEU - Int. J. Electron. Commun., vol. 70, no. 7, pp. 910-919, Jul. 2016.

[18] R. A. Sadeghzadeh, M. Alibakhshi-Kenari, and M. Naser-Moghadasi, "UWB antenna based on SCRLH-TLs for portable wireless devices," Microw. Opt. Technol. Lett., vol. 58, no. 1, pp. 69-71, Jan. 2016.

[19] S. Otto, A. Al-Bassam, A. Rennings, K. Solbach, and C. Caloz, "Transversal asymmetry in periodic leaky-wave antennas for bloch impedance and radiation efficiency equalization through broadside," IEEE Trans. Antennas Propag., vol. 62, no. 10, pp. 5037-5054, 2014.

[20] S. Otto, Z. Chen, A. Al-Bassam, A. Rennings, K. Solbach, and C. Caloz, "Circular polarization of periodic leaky-wave antennas with axial asymmetry: theoretical proof and experimental demonstration," IEEE Trans. Antennas Propag., vol. 62, no. 4, pp. 1817-1829, 2014.

[21] Q. Zhang, D. L. Sounas, S. Gupta, and C. Caloz, "Wave-interference explanation of group-delay dispersion in resonators [Education Column]," IEEE Antennas Propag. Mag., vol. 55, no. 2, pp. 212227, 2013.

[22] M. Alibakhshi-Kenari, M. Naser-Moghadasi, R. A. Sadeghzadeh, B. S. Virdee, and E. Limiti, "Dualband RFID tag antenna based on the Hilbert-curve fractal for HF and UHF applications," IET Circuits, Devices Syst., vol. 10, no. 2, pp. 140-146, 2016.

[23] S. Gupta, Q. Zhang, L. Zou, L. J. Jiang, and C. Caloz, "Generalized coupled-line all-pass phasers," IEEE Trans. Microw. Theory Tech., vol. 63, no. 3, pp. 1007-1018, 2015.

[24] D. L. Sounas, T. Kodera, and C. Caloz, "Electromagnetic modeling of a magnetless nonreciprocal gyrotropic metasurface," IEEE Trans. Antennas Propag., vol. 61, no. 1, pp. 221-231, 2013. 
[25] H. Chen and C. T. Chan, "Acoustic cloaking and transformation acoustics," J. Phys. D: Applied Physics, vol. 43, no. 11, pp. 113001, 2010.

[26] F. Sun and S. He, "Remote cooling by a novel thermal lens with anisotropic positive thermal conductivity," Sci. Rep, vol. 7, no. 1, 2017.

[27] V. Veselago, L. Braginsky, V. Shklover, and C. Hafner, "Negative refractive index materials," J. Comput. Theor. Nanosci., vol. 3, pp. 189-218, 2006.

[28] W. Li, F. Meng, Y. Chen, Y. fan Li, and X. Huang, "Topology optimization of photonic and phononic crystals and metamaterials: A review," Adv. Theory Simulations, vol. 2, no. 7, p. 1900017, 2019.

[29] D.-H. Kwon and D. H. Werner, "Transformation optical designs for wave collimators, flat lenses and right-angle bends," New J. Phys., vol. 10, no. 11, pp. 115023, 2008.

[30] D.-H. Kwon and D. H. Werner, "Flat focusing lens designs having minimized reflection based on coordinate transformation techniques," Opt Express, vol. 17, no. 10, pp. 7807, 2009.

[31] P. Zhang, "Theory of transformation optics and invisibility cloak design," Ph.D. thesis, Stockholm, KTH Royal Institute of Technology, 2011.

[32] J. Hu, X. Zhou, and G. Hu, "Design method for electromagnetic cloak with arbitrary shapes based on Laplace's equation," Opt. Express, vol. 17, no. 3, pp. 1308, 2009.

[33] Z. Chang, X. Zhou, J. Hu, and G. Hu, "Design method for quasi-isotropic transformation materials based on inverse Laplace's equation with sliding boundaries," Opt Express, vol. 18, no. 6, pp. 6089-96, 2010.

[34] J.-J. Ma, X.-Y. Cao, K.-M. Yu, and T. Liu, "Determination the material parameters for arbitrary cloak based on Poisson's equation," Prog. Electromagn. Res M, vol. 9, pp. 177-184, 2009.

[35] X. Chen, Y. Fu, and N. Yuan, "Invisible cloak design with controlled constitutive parameters and arbitrary shaped boundaries through Helmholtz's equation," Opt Express, vol. 17, no. 5, pp. 3581-6, 2009.

[36] D. Schurig, J. J. Mock, B. J. Justice, S. A. Cummer, J. B. Pendry, A. F. Starr, and D.R. Smith, "Metamaterial electromagnetic cloak at microwave frequencies," Science, vol. 314, no. 5801, pp. 977-80, Nov 10, 2006.

[37] D.-H. Kwon and D. H. Werner, "Transformation optical designs for wave collimators, flat lenses and right-angle bends," New J. Phys., vol. 10, no. 11, pp. 115023, 2008.

[38] J. Hu, X. Zhou, and G. Hu, "Design method for electromagnetic cloak with arbitrary shapes based on Laplace's equation," Opt Express, vol. 17, no. 3, pp. 1308, 2009.

[39] Z. Chang, X. Zhou, J. Hu, and G. Hu, "Design method for quasi-isotropic transformation materials based on inverse Laplace's equation with sliding boundaries," Opt Express, vol. 18, no. 6, pp. 6089-96, 2010.

[40] J.-J. Ma, X.-Y. Cao, K.-M. Yu, and T. Liu, "Determination the material parameters for arbitrary cloak based on Poisson's equation," Progress in Electromagnetics Research M, vol. 9, pp. 177-184, 2009.

[41] X. Chen, Y. Fu, and N. Yuan, "Invisible cloak design with controlled constitutive parameters and arbitrary shaped boundaries through Helmholtz's equation," Opt Express, vol. 17, no. 5, pp. 3581-6, 2009.

[42] E. A. Berry, J. J. Gutierrez, and R. C. Rumpf, "Design and simulation of arbitrarily-shaped transformation optic devices using a simple finite-difference method," Prog. Electromagn. Res B, vol. 68, pp. 1-16, 2016.

[43] R. C. Rumpf, C. R. Garcia, E. A. Berry, and J. H. Barton, "Finite-difference frequency-domain algorithm for modeling electromagnetic scattering from general anisotropic objects," Prog. Electromagn. Res B, vol. 61, pp. 55-67, 2014.

[44] R. C. Rumpf, "Simple implementation of arbitrarily shaped total-field/scattered-field regions in finitedifference frequency-domain," Prog. Electromagn. Res B, vol. 36, pp. 221-248, 2012.

[45] Y. Liu, F. Sun, and S. He, "Controlling lightwave in Riemann space by merging geometrical optics with transformation optics," Sci Rep, vol. 8, no. 1, pp. 514, 2018.

[46] P. Jarutatsanangkoon, W. S. Mohammed, and W. Pijitrojana, "Transformation optics based on unitary vectors and Fermat's principle for arbitrary spatial transformation design," Appl. Opt, vol. 57, no. 29, pp. 8632, 2018. 